\title{
Ethnische Vielfalt und Arbeitsmarkterfolg
}

\author{
Ulf Rinne • Simone Schüller · Klaus F. Zimmermann
}

Angenommen: 25. Januar 2011 / Online publiziert: 9. Februar 2011

(C) Institut für Arbeitsmarkt- und Berufsforschung 2011

Zusammenfassung Das ökonomische Potenzial ethnischer und kultureller Vielfalt wird häufig verkannt. Die Ergebnisse neuerer Studien, die in diesem Beitrag zusammengefasst werden, zeigen jedoch, dass sich „weiche“ Faktoren wie etwa Einstellungen, Wahrnehmungen und Identitäten, hier insbesondere ethnische Identitäten, wesentlich auf ökonomische Ergebnisse auswirken können. Dies geht sowohl aus Analysen des Prozesses kultureller Integration in einer generationenübergreifenden Perspektive als auch aus Untersuchungen der Arbeitsplatzsuche und der Wiedereingliederung von Arbeitslosen in den Arbeitsmarkt hervor. Eine Volkswirtschaft kann sich durch eine geeignete Beachtung und Einbeziehung multi-ethnischer Faktoren ökonomisch besser stellen. Kulturelle und ethnische Assimilation von Zuwanderern ist deshalb keine alleinige oder dominante Strategie der ökonomisch erfolgreichen Einbindung in die Aufnahmegesellschaft. Neben einer besseren Aktivierung der Integrationspotenziale bei Personen mit Migrationshintergrund ist auch eine kulturelle oder ethnische Öffnung der Einheimischen sinnvoll.

Schlüsselwörter Migration · Ethnicity · Ethnic identity · Labor Market Success

JEL Klassifikationen F22 · J15 · J61

Ethnic diversity and labor market success

Abstract The economic potential of ethnic and cultural diversity is often underesti-mated. This paper summarizes a number of recent studies which show that "soft" factors such as attitudes, perceptions and identities-and in particular ethnic identities-significantly affect economic outcomes. More specifically, the studies analyze the process of cultural integration over migrant genera-tions as well as the process of job search and labor market reintegration of the unemployed. The economy can thus gain productivity and efficiency by recognizing and incorporating such multi-ethnic factors. Cultural assimilation that goes along with a loss of migrants' own cultural heritage does not appear to be the sole or dominant strategy of an economically successful integration. To tap the full potential of ethnic and cultural diversity, an increased cultural and ethnic open-mindedness of the native population is desirable.

\section{Einleitung}

Ethnische und kulturelle Vielfalt stellen aus ökonomischer Sicht die Basis für ein Potenzial wirtschaftlichen Erfolges und Wohlstandes dar, das häufig verkannt wird. In einer zunehmend globalisierten und hochgradig arbeitsteiligen Gesellschaft stellt ethnisches Humankapital von Immigranten einen ökonomisch wertvollen Faktor dar, der wirtschaftliche Dynamik und Kreativität zu fördern vermag (vgl. Alesina und La Ferrara 2005). Ethnische Vielfalt und kulturelle Diversität sind allerdings in der gesellschaftlichen Diskussion häufig Ausgangspunkte von wirtschaftlichen Verdrän- 
gungsängsten und Überfremdungsgefühlen. Problematisch erscheint auch die oft schlechte wirtschaftliche Performance von Migranten, die vordergründig gegen die Ausweitung von Zuwanderung spricht.

Diese kritische Diskussion ist problematisch, weil Immigranten so das Gefühl vermittelt wird, im Zielland nicht willkommen zu sein, was ihre Integrationsbereitschaft hemmen kann. Neben der grundsätzlichen Frage nach den Vorteilen von Einwanderung für eine Volkswirtschaft rückt deshalb die Frage in das Zentrum der Aufmerksamkeit, inwiefern und inwieweit die Bindung oder Identifikation der Immigranten mit den Heimat- und Aufnahmeländern selbst ein wichtiger Faktor für den ökonomischen Erfolg der Migranten und der beteiligten Länder ist. Der Prozess von Anpassung und Integration, den Immigranten nach ihrer Ankunft im Bestimmungsland durchlaufen, und damit ihre faktische multi-ethnische Identität, trägt in nicht unerheblichem Maße dazu bei, die Wirkungen von ethnischer Diversität auf wirtschaftliche Entwicklung und Arbeitsmarkterfolg zu gestalten.

Immigranten werden typischerweise zunächst mit Einheimischen verglichen. Diese Vergleiche zeigen für Europa, dass die durchschnittlichen Arbeitsmarkterfolge von Immigranten hinter denen der Einheimischen zurückbleiben (vgl. u.a. Kahanec und Zaiceva 2009; Kahanec und Zimmermann 2010). Die Gründe hierfür sind vielfältig: Sie reichen von einer nicht entsprechenden Humankapitalausstattung (Kalter und Granato 2007) über mangelnde Sprachkenntnisse und Diskriminierung (Dustmann und Fabbri 2003; Kaas und Manger 2010) bis hin zu zuwandererspezifischen Marktsegmenten, die geringere Einkommen und Aufstiegschancen bieten (Heath und Li 2008). Dennoch besitzen Immigranten zweifelsohne spezielle Fähigkeiten, über die Einheimische nicht verfügen. Die Aktivierung dieser vielfach komplementären Qualifikationen (z.B. Problemlösungskompetenzen und -ansätze, Kreativität oder Adaptationsfähigkeit, vgl. Ottaviano und Peri 2006) ist die zentrale Voraussetzung dafür, dass Wirtschaft und Gesellschaft zugunsten eines gröBeren Wohlstandes insgesamt von Zuwanderung profitieren. Bisherige Forschungsergebnisse deuten darauf hin, dass ethnische Identitäten ${ }^{1}$ hierbei eine zentrale Bedeutung haben. Es zeigt sich zudem, dass multiple ethnische Identitäten kein Hindernis, sondern vielmehr Garant eines wirtschaftlichen Erfolges sein können.

Darauf aufbauend ist es Zielsetzung der Forschungsaktivitäten im Rahmen des Projektes „Ethnische Vielfalt

\footnotetext{
${ }^{1}$ Akerlof und Kranton (2000) definieren Identität im Rahmen der ökonomischen Theorie als das individuelle Selbstbild einer Person (,,a person's sense of self"). Individuen ziehen einen großen Nutzen aus diesem Selbstbild entsprechendem Verhalten, weshalb die Identitätswahl in erheblichem Maße ökonomische Entscheidungen beeinflussen kann. Ethnische Identität ist der Teil des Selbstkonzeptes, der sich aus dem Wissen um Gruppenzugehörigkeit und dessen emotionalem Wert ergibt (Tajifel 1981).
}

und Arbeitsmarkterfolg“, die empirische Analyse ethnischer Identität als Determinante ökonomischen Erfolges auf hauptsächlich zwei Fragenkomplexe auszuweiten: Zum einen wird der Frage nachgegangen, wie sich der Prozess kultureller Integration aus einer generationenübergreifenden Perspektive darstellt und welche Auswirkungen sich auf den ökonomische Erfolg der zweiten Immigrantengeneration erkennen lassen. Dabei stützt sich das Projekt auf das bewährte Datenmaterial des Sozio-ökonomischen Panels (SOEP) des DIW Berlin, das bereits Grundlage vieler Studien zu den ökonomischen Konsequenzen ethnischer Diversität war (Constant et al. 2009c; Constant und Zimmermann 2008, 2009). Zum Anderen bietet der IZA Evaluationsdatensatz (Caliendo et al. 2010) die Möglichkeit, die Analyse ethnischer Identität auf Aspekte der Arbeitsplatzsuche und der Wiedereingliederung von Arbeitslosen in den Arbeitsmarkt zu erweitern. Beide Themenkomplexe behandeln zentrale, jedoch im deutschen Kontext derzeit noch weitgehend unerforschte Fragestellungen.

Die Integration der in Deutschland geborenen Personen mit Migrationshintergrund, der sogenannten zweiten Generation, gewinnt zunehmend an politischer und ökonomischer Relevanz. Heute haben bereits ein Drittel der Kinder im Alter von bis zu fünf Jahren einen Migrationshintergrund (Rühl 2009), was die zukünftige Bedeutung dieser Gruppe für den deutschen Arbeitsmarkt verdeutlicht. In Bezug auf Integrationspolitik stellt der Grad, in dem sie Bildungsund Chancengleichheit erfährt, den vielleicht wichtigsten Erfolgsindikator dar. Betrachtet man ökonomische Ergebnisvariablen, wie z.B. Bildungsniveau und Arbeitsmarkterfolge, so findet man jedoch in Deutschland einen beständigen Nachteil beider Generationen von Einwanderern gegenüber den Einheimischen (vgl. Algan et al. 2010). Die Erwartung, dass sich dieser Nachteil von der ersten zur zweiten Generation der Immigranten reduziert, findet somit keine Bestätigung. Die ökonomische Integration der zweiten Generation scheint nicht zu gelingen.

Es stellt sich daher die Frage, ob dies (auch) auf eine mangelnde kulturelle Integration oder eine abweichende, nicht konvergierende ethnische Identität zurückzuführen ist. Die nachfolgend beschriebenen Studien, die sich der Beantwortung dieses Fragenkomplexes widmen, nutzen das Sozio-ökonomische Panel (SOEP), eine repräsentative Wiederholungsbefragung privater Haushalte in Deutschland. Das SOEP wird bereits seit 25 Jahren im Auftrag des DIW Berlin durchgeführt und beinhaltet die gegenwärtig größte Wiederholungsbefragung von in Deutschland lebenden Ausländern. Insbesondere Haushalte mit einem Haushaltsvorstand türkischer, spanischer, italienischer, griechischer und ehemals jugoslawischer Herkunft sind überproportional repräsentiert. Die Daten geben Auskunft zu Fragen über Einkommen, Erwerbstätigkeit, Bildung oder Gesundheit. Einwanderungsspezifische Fragestellungen bein- 
halten unter anderem Aspekte der Sprachverwendung, des Kontakts mit Einheimischen sowie der Identifikation mit der deutschen Kultur bzw. der des Heimatlandes.

Zur Analyse der Auswirkungen ethnischer Identität auf Aspekte der Arbeitsplatzsuche und der Wiedereingliederung von Arbeitslosen in den Arbeitsmarkt wird der IZA Evaluationsdatensatz genutzt (Caliendo et al. 2010). Dieser neue Datensatz besteht aus zwei Säulen: Verwaltungsdaten der Bundesagentur für Arbeit sowie Befragungsdaten des IZA. Die nachfolgenden Studien stützen sich auf eine Befragung von rund 18.000 Personen, die sich zwischen Juni 2007 und Mai 2008 arbeitslos gemeldet haben. Einer der zahlreichen Vorteile des Datensatzes besteht darin, dass die Individuen bereits sehr zeitnah nach ihrem Eintritt in die Arbeitslosigkeit interviewt wurden. Weitere Interviews finden jeweils ein Jahr und drei Jahre später statt. Darüber hinaus beinhaltet das Fragenspektrum eine Vielzahl von Themen, die immer noch recht selten in derartigen Befragungen erhoben werden, aber deren Einfluss auf ökonomische Ergebnisvariablen mehr und mehr in das Zentrum der Aufmerksamkeit rückt. Dies betrifft etwa Persönlichkeitsmerkmale, Einstellungen und kognitive Fähigkeiten. Daneben werden detaillierte Informationen zum Migrationshintergrund und zur ethnischen Identität erhoben. So bietet der IZA Evaluationsdatensatz die Gelegenheit, den Einfluss dieses reichhaltigen Fragenspektrums unter dem speziellen Gesichtspunkt der Arbeitsplatzsuche und der Wiedereingliederung von Arbeitslosen in den Arbeitsmarkt zu studieren.

Nachfolgend werden die zentralen Ergebnisse und Implikationen unseres Projektes vorgestellt, das die Auswirkungen des kulturellen Integrationsprozesses von Personen mit Migrationshintergrund in Deutschland auf deren Arbeitsmarkterfolg untersucht. Zunächst wird der bisherige Forschungsstand sowie das zugrunde liegende Konzept ethnischer Identität vorgestellt. Im Folgenden werden Befunde hinsichtlich des generationenübergreifenden Integrationsprozesses diskutiert, bevor sich der darauf folgende Abschnitt genauer mit den Auswirkungen ethnischer Identität auf den Prozess der Arbeitsplatzsuche auseinandersetzt. Der Beitrag schließt mit einem Fazit und Ausblick.

\section{Bisheriger Forschungsstand und Messung von ethnischer Identität}

In ihrem Handbuchartikel erfassen Constant und Zimmermann (2011) zentrale Forschungsergebnisse der bisherigen Migrationsforschung. Sie zeigen dabei, dass neben Ethnizität oder ethnischer Herkunft ,weiche“ Faktoren wie etwa Einstellungen, Wahrnehmungen und Identitäten als Ein- flussfaktoren des ökonomischen Erfolges von Immigranten zunehmendes Interesse innerhalb der jüngeren ökonomischen Forschung erlangen. ${ }^{2}$ In der bisherigen empirischen Forschung stellen sich derartige Faktoren als faktisch exogener Natur für den ökonomischen Prozess dar, d.h. sie scheinen ökonomische Entscheidungen in einem weit stärkeren Ausmaß zu beeinflussen, als dass sie selbst durch ökonomische Faktoren beeinflusst werden (Constant und Zimmermann 2008). Es zeigt sich zudem, dass ethnische Identität und Arbeitsmarkterfolg hauptsächlich über den Zugang zum Erwerbsleben in Bezug stehen, während kein signifikanter Zusammenhang zwischen ethnischer Identität und individuellem Einkommen nachgewiesen werden konnte (Constant und Zimmermann 2009).

Des Weiteren wird deutlich, dass sich das Streben nach einer vollständigen Assimilation der Immigranten nicht ausschließlich mit ökonomischer Rationalität begründen lässt. Insbesondere für Immigranten der ersten Generation ist eine vollständige Assimilation praktisch ausgeschlossen. Assimilation und Integration sind demnach hauptsächlich als generationenübergreifende Prozesse zu verstehen. Zudem ist es gerade ihre Verschiedenheit bzw. der Umstand, dass Immigranten im Zielland benötigte Charakteristika haben, weshalb eine Zuwanderung sinnvoll ist und diese zustande kommt. Eine Beseitigung dieser Unterschiede würde auch die Vorteile zum Verschwinden bringen, die durch kulturelle Diversität ausgelöst oder doch zumindest begünstigt werden (vgl. Bellini et al. 2009; Audretsch et al. 2008). Der Befund, dass Assimilation und Integration ökonomische Ergebnisvariablen positiv beeinflussen, während Effekte von Separierung und Marginalisierung negativ sind, unterstützt diese Sichtweise. ${ }^{3}$ Eine integrierte, also multi-ethnische Identität scheint demnach kein Hindernis ökonomischen Erfolges zu sein, sondern sie ist vielmehr in Hinblick auf eine erfolgreiche Arbeitsmarktintegration von Immigranten wünschenswert.

Constant und Zimmermann (2011) argumentieren, dass es als Folge von demographischem Wandel und Globalisierung unausweichlich zu vermehrten weltweiten arbeitsmarktorientierten Migrationsströmen und auf nationaler Ebene zu größeren ethnischen Diversitäten kommen wird. Das Verständnis der Herausbildung multi-ethnischer Identitäten und des Zusammenspiels von kultureller Tradition,

\footnotetext{
${ }^{2}$ Dokumentiert wird dieses steigende Interesse beispielsweise anhand von Sonderausgaben des Journal of Population Economics (Volume 20, Issue 3, 2007), des International Journal of Manpower (Volume 30, Issue 1-2, 2009), Research in Labor Economics (Volume 29, 2009) oder kürzlich The Economic Journal (Volume 120, Issue 542).

${ }^{3}$ Eine genauere Erläuterung der vier Zustände der Identitätsfindung von Immigranten, die hier genannt werden, findet sich weiter unten in diesem Text.
} 
Diversität und deren Wahrnehmung innerhalb verschiedener Bevölkerungsgruppen ist daher von höchster ökonomischer und insbesondere politischer Relevanz.

Der Integrationsprozess von Immigranten in Deutschland lässt sich anhand des Grades verdeutlichen, mit dem die Identifikation mit dem Aufnahmeland zunimmt. Mit dem Eintreffen im Bestimmungsland werden Immigranten mit gesellschaftlichen und kulturellen Normen konfrontiert, die von der Kultur ihres Heimatlandes abweichen. In der neuen Heimat hat dies auch potenziell Auswirkungen auf das ursprüngliche Zugehörigkeitsempfinden zum Herkunftsland. Im Extremfall wird dabei die Identifikation mit der Ursprungskultur vollständig aufgegeben oder eine Bindung an die Kultur und Gesellschaft des Aufnahmelandes bleibt restlos aus. Dazwischen sind weitere Abstufungen der Identitätsfindung von Immigranten denkbar. Insgesamt lassen sich folgende Zustände unterscheiden (vgl. Constant et al. 2009a):

- Assimilation: starke Verbundenheit mit dem Aufnahmeland bei nur noch schwacher Bindung an das Herkunftsland;

- Integration: starke Identifikation mit Kultur und Gesellschaft des Aufnahmelandes bei gleichzeitig starkem $\mathrm{Zu}$ gehörigkeitsgefühl zu dem Herkunftsland;

- Separation: Identifikation ausschließlich mit der Ursprungskultur;

- Marginalisierung: keine Bindung, weder an die Kultur des Aufnahmelandes noch zu derjenigen des Herkunftslandes.

In ähnlicher Weise können auch Einheimische einen Prozess der Neufindung eigener ethnischer Identität durchlaufen, wenn sie mit anderen Kulturen konfrontiert sind. Man kann dies als Grad kultureller Offenheit verstehen, der in einer globalisierten Welt ökonomisch, politisch und gesellschaftlich immer bedeutsamer wird. Dabei wird deutlich, dass in langfristiger und historischer Perspektive auch Kultur selbst prinzipiell kein statisches oder festes Gebilde ist, sondern sich mit der Aufnahme von Elementen anderer Kulturen weiterentwickelt. Assimilation heißt deshalb - radikal zu Ende gedacht - zunächst Aufgabe der eigenen ethnischen Identität zu Gunsten einer festen ethnischen Identität des Aufnahmelandes. Langfristig werden aber integrierte Migranten, also solche, die sich auch die Kultur ihres Heimatlandes bewahrt haben, ihre kulturellen Errungenschaften in die Kultur des Aufnahmelandes einbringen. Was deutsch war und ist, ist also in einem langfristigen Wandel begriffen. Wir beschränken uns in diesem Projekt allerdings auf kurzfristige Anpassungsprozesse und lassen die Frage der dynamischen Entwicklung von „Kultur“ offen.

\section{Ethnische Identität: Zwischen Assimilation, Integration und Vielfalt}

Constant et al. (2011) untersuchen den kulturellen Integrationsprozess von Immigranten in Deutschland genauer. ${ }^{4} \mathrm{Ne}-$ ben einem Vergleich mit der einheimischen Bevölkerung werden auch Einwanderer der ersten und zweiten Generation miteinander verglichen. ${ }^{5}$ Indikatoren, anhand derer sie die verschiedenen Integrationsprozesse analysieren, beinhalten neben dem Familienstand, Sprachfähigkeiten, der ethnischen Identifikation und der Religiosität auch Faktoren wie Lebenszufriedenheit, Risikoaversion und politisches Interesse. Insgesamt zeigt sich, dass sowohl zwischen Immigranten und Einheimischen als auch zwischen den zwei Generationen von Immigranten substanzielle Unterschiede hinsichtlich ihrer Integration existieren, welche zudem nach Herkunft und Geschlecht signifikant unterschiedlich ausfallen. $^{6}$

Immigranten der zweiten Generation zeigen im Vergleich $\mathrm{zu}$ ihrer Elterngeneration eine größere kulturelle, sozioökonomische und politische Integration in die deutsche Gesellschaft, was auf einen erfolgreichen intergenerationalen Integrationsprozess hinweist. Beispielsweise unterscheiden sich die späteren Generationen in Bezug auf Heiratsverhalten sowie Fertilität nicht mehr signifikant von der deutschen Mehrheitsbevölkerung, während die erste MigrantenGeneration dahingehend noch deutliche Unterschiede aufweist. ${ }^{7}$ Frauen der zweiten Generation weisen meist eine höhere sprachliche Anpassung auf als Männer. Auch sind besonders Frauen griechischer und türkischer Herkunft hinsichtlich interethnischen Heiratsverhaltens zurückhaltender als Männer dieser Herkunftsgruppen.

Bezüglich der Indikatoren ,ethnische Identifikation“ und „politisches Interesse“ weisen Einwanderer polnischer und russischer Herkunft eine vergleichsweise starke Integration auf. Dies ist womöglich auf den hohen Anteil ethnisch deut-

\footnotetext{
${ }^{4}$ Sie verwenden zu diesem Zweck das SOEP der Jahre 2005 bis 2007.

${ }^{5}$ Die Definition von Immigranten zweiter Generation kann sich zwischen den Studien unterscheiden. Während Constant et al. (2011) sowie Constant et al. (2010a) auch Immigranten zweiter Generation einschließen, bei denen ein Elternteil deutscher Herkunft ist, sind solche Fälle in Schüller (2010) sowie Krause et al. (2010) von der Analyse ausgeschlossen. Hier werden ausschließlich Personen betrachtet, deren beide Elternteile einen Migrationshintergrund aufweisen.

${ }^{6}$ Die Ergebnisse beruhen auf Pooled OLS sowie Logit Schätzungen, anhand derer die jeweiligen Indikatoren kultureller Integration auf Indikatorvariablen für erste und zweite Migrantengeneration, interagiert mit der ethnischen Gruppenzugehörigkeit, regressiert werden. Regressionsmodelle werden separat für Frauen und Männer geschätzt und es wird jeweils für verschiedene Geburtskohorten sowie das Bildungsniveau kontrolliert.

${ }^{7}$ Immigranten erster Generation weisen im Vergleich zur ethnisch deutschen Bevölkerung und der zweiten Generation eine höhere Heiratstendenz, eine höhere Fertilität und ein jüngeres Heiratsalter auf.
} 
scher Einwanderer in diesen Herkunftsgruppen zurückzuführen. Die Befunde für türkische Einwanderer sind insgesamt wenig ermutigend, denn sie zeigen die geringste Integration von allen ethnischen Einwandergruppen in Deutschland, insbesondere im Bereich sprachlicher Fähigkeiten und ethnischer Identifikation. Dennoch ist auch für diese Gruppe ein positiver Trend im Zeitverlauf in Richtung einer stärkeren Anpassung erkennbar.

Es zeigt sich also bezüglich der kulturellen Integration ein insgesamt positiver Trend im Zeitverlauf und über die Generationen von Einwanderern hinweg. Dessen ungeachtet bleibt ein persistenter Nachteil beider Generationen gegenüber Einheimischen in Deutschland im Hinblick auf ökonomische Ergebnisvariablen bestehen (vgl. Algan et al. 2010).

Die Studie von Schüller (2010) geht weiterführend der Frage nach, wie sich die Identifikation von Zuwandererfamilien mit dem Heimat- bzw. Aufnahmeland im generationenübergreifenden Prozess der elterlichen Bildungsinvestitionen widerspiegelt. Kinder mit Migrationshintergrund schlagen in Deutschland vielfach niedrigere Bildungsgänge ein und sind in Hauptschulen überrepräsentiert (vgl. auch Riphahn 2005). Dies ist hauptsächlich durch grundlegende Unterschiede des sozioökonomischen Familienhintergrundes zwischen Kindern mit und ohne Migrationshintergrund zu erklären (Krause et al. 2010).

Es könnte jedoch Unterschiede innerhalb der Zuwandererbevölkerung geben. Schüller (2010) untersucht, ob Eltern, die besser in die deutsche Gesellschaft integriert sind bzw. eine starke Bindung an die Kultur ihres Herkunftslandes beibehalten, den Schulerfolg ihrer Kinder mehr oder weniger erfolgreich fördern können. Geht man davon aus, dass Eltern ihre kulturelle Identität in die Kindererziehung einfließen lassen (Bisin und Verdier 2001), so ergibt sich ein theoretischer Zusammenhang zwischen dem Grad elterlicher Identifikation mit dem Aufnahme- bzw. Herkunftsland und dem Bildungserfolg ihrer Nachkommen.

Um diesen Zusammenhang empirisch zu untersuchen, betrachtet die Studie den ersten Bildungsübergang von Kindern mit Migrationshintergrund im deutschen Schulsystem von der Grundschule in weiterführende Schulen. Empirische Schätzungen ${ }^{8}$ ergeben, dass beide Dimensionen elterlicher Identität - die Bindung an das Herkunftsland sowie die Identifikation mit der deutschen Gesellschaft - in einem positiven Zusammenhang mit der Wahrscheinlichkeit stehen, einen höheren Bildungsweg einzuschlagen. Dabei sind es die Mütter, deren Hinwendung zum Aufnahmeland bedeutend ist, während in Bezug auf die Väter die relativ stär-

\footnotetext{
${ }^{8}$ Empirische Schätzungen erfolgen anhand von Probit Regressionen mit Übergangswahrscheinlichkeit auf eine höhere Schulform (Realschule oder Gymnasium) als abhängige Variable. Kontrolliert wird u.a. für Bildung der Eltern, sozioökonomischen Hintergrund der Familie, Herkunftsland, sowie Länge des Aufenthaltes in Deutschland.
}

kere Verbundenheit zum Heimatland einen signifikant positiven Effekt aufweist. ${ }^{9}$ Dies könnte darauf hinweisen, dass eine kulturelle Integration von Migranten in Bezug auf den ökonomischen Erfolg zukünftiger Generationen mit Migrationshintergrund wünschenswert ist, wobei die Beibehaltung einer Bindung an das Herkunftsland keinesfalls hinderlich sein muss - im Gegenteil, diese kann einer ökonomischen Integration ebenfalls zuträglich sein.

\section{Ethnische Vielfalt im Suchprozess}

Weitere Untersuchungen haben den Prozess der Arbeitsplatzsuche von Einwanderern der ersten und zweiten Generation im Vergleich mit Einheimischen analysiert. Dieser Prozess ist ein bedeutender ökonomischer Mechanismus für den individuellen Arbeitsmarkterfolg, dessen Relevanz zugenommen hat und weiter zunimmt: Gebrochene Erwerbsbiographien prägen längst die Realität auf dem deutschen Arbeitsmarkt, das Normalarbeitsverhältnis befindet sich auf dem Rückzug und atypische Beschäftigungsverhältnisse erleben einen Aufschwung (vgl. Eichhorst et al. 2010). Vor diesem Hintergrund sind zumindest kürzere Phasen der Arbeitslosigkeit und der Arbeitsplatzsuche eher die Regel als die Ausnahme. Damit steigt auch die Relevanz der Frage, inwieweit sich Personen mit Migrationshintergrund und Einheimische in dieser Hinsicht unterscheiden: Bestehen diesbezüglich bedeutende Unterschiede, kann (und sollte) etwa die Ausgestaltung der aktiven Arbeitsmarktpolitik diese berücksichtigen.

So zeigt die Untersuchung des Prozesses der Arbeitssuche in Constant et al. (2009b), dass ethnische Identität und der Grad der Integration in diesem Kontext eine wesentliche Rolle spielen. Die Ergebnisse verdeutlichen, dass Strategien einer frühzeitigen Intervention nach dem Eintritt in die Arbeitslosigkeit insbesondere auf die Gruppe separierter Immigranten abzielen sollte. Diese Gruppe wird als diejenige mit besonderen Schwierigkeiten im Suchprozess bzw. bei der Reintegration in reguläre Beschäftigung identifiziert. Vergleichsweise hohe Reservationslöhne scheinen hierbei eine wichtige Rolle zu spielen.

Aus diesem Grund werden diese Untersuchungen in Constant et al. (2010a) um eine genauere Betrachtung der Reservationslöhne erweitert. Dabei zeigt sich, dass es aufgrund verschiedener Referenzgruppen zu unterschiedlich hohen Anspruchslöhnen kommen kann. Unter der Annahme, dass

\footnotetext{
${ }^{9}$ Schätzungen ergeben einen signifikanten durchschnittlichen marginalen Effekt der mütterlichen deutschen Identität auf die Übergangswahrscheinlichkeit auf eine höhere Schulform je nach Modell-Spezifikation von ca. 5-7 Prozent, und einen signifikanten Effekt väterlicher Herkunftsidentität in ähnlicher Größenordnung.
} 
sich Immigranten der ersten Generation bei ihren Reservationslöhnen zu einem stärkeren Ausmaß an ihrem Ursprungsland bzw. an Immigranten aus diesem Land orientieren und Einwanderer der zweiten Generation und Einheimische ihre Ansprüche in erster Linie bzw. ausschließlich am Lohnniveau in Deutschland ausrichten, resultieren für letztere Gruppen ceteris paribus höhere Reservationslöhne. ${ }^{10}$ Empirisch kann diese Hypothese bestätigt werden: Für die zweite Generation von Einwanderern ergeben sich signifikant höhere Reservationslöhne, die im Vergleich mit Immigranten der ersten Generation um rund 3.5 Prozent höher ausfallen, auch wenn für beobachtbare Charakteristika kontrolliert wird. Eine Blinder-Oaxaca Dekomposition gibt zudem Hinweise darauf, dass die zweite Generation für gleiche Charakteristika eine höhere Entlohnung erwartet. Dies betrifft unter anderem die erwarteten Bildungsrenditen.

Constant et al. (2010b) analysieren Präferenzen und Einstellungen von arbeitslosen Einheimischen und Einwanderern der zweiten Generation. Es werden Risikopräferenzen, Zeitpräferenzen, Vertrauen und Reziprozität untersucht. Neuere Forschungsergebnisse belegen, dass sich derartige Faktoren in ökonomischen Ergebnisvariablen niederschlagen und diese wesentlich beeinflussen (vgl. etwa Borghans et al. 2008). Unter anderem wirken sich solche Merkmale auch auf den Prozess der Arbeitsplatzsuche aus. Das Wissen um diesbezügliche etwaige Unterschiede zwischen den betrachteten Personengruppen kann deshalb ausgesprochen hilfreich sein, wenn es zum Beispiel um eine adäquate Gestaltung arbeitsmarktpolitischer Interventionen geht. In der Untersuchung zeigen sich signifikante Unterschiede zwischen arbeitslosen Einheimischen und Einwanderern der zweiten Generation hinsichtlich ihrer Risikopräferenzen und des Grades der positiven Reziprozität. Letzteres misst den Grad, zu dem eine Person bereit ist, auf ein großzügiges, faires oder freundliches Verhalten entsprechend positiv zu reagieren. Bereits nach kurzer Zeit der Arbeitsplatzsuche erklärt zudem die geringere Risikoaversion von Personen mit Migrationshintergrund zumindest teilweise die geringere Reintegrationswahrscheinlichkeit dieser Gruppe im Vergleich zu den Einheimischen. Ein möglicher Mechanismus, der diesen Effekt hervorruft, liegt in höheren Reservationslöhnen, die sich für Personen mit höherer Risikofreude ergeben (vgl. Pannenberg 2010).

\section{Fazit und Ausblick}

Die hier vorgestellten Studien zeigen, dass sich ethnische Identität nachhaltig, d.h. über Generationen hinweg, auf

\footnotetext{
${ }^{10}$ Es wird darüber hinaus angenommen, dass das Lohnniveau im Ursprungsland bzw. dasjenige von Immigranten aus diesem Land geringer als das (durchschnittliche) Lohnniveau in Deutschland ausfällt.
}

ökonomische Ergebnisvariablen auswirkt. Sie liefern darüber hinaus erste Erkenntnisse bezüglich der Auswirkungen auf den Bildungserfolg sowie die Reservationslöhne der zweiten Generation.

Zunächst kann festgestellt werden, dass der Prozess kultureller Integration von Einwanderern in Deutschland, gemessen in Generationen, innerhalb aller ethnischen Einwanderergruppen stattfindet. Beispielsweise bezüglich Heiratsund Fertilitätsverhalten, Risikoaversion oder politischem Interesse unterscheiden sich in Deutschland geborene Kinder von Einwanderern kaum mehr von der einheimischen Bevölkerung. Diese kulturelle Annäherung zeigt sich - je nach kultureller Distanz - für einzelne Einwanderergruppen mehr oder weniger deutlich. Der insgesamt positive generationenübergreifende Trend ist jedoch für jede einzelne Gruppe evident.

Die Ergebnisse der in diesem Beitrag vorgestellten Studien bestätigen ferner, dass sich ,weiche“ Faktoren wie etwa Einstellungen, Wahrnehmungen und ethnische Identitäten wesentlich auf ökonomische Ergebnisvariablen auswirken. Es kann zudem gezeigt werden, dass dies ein nachhaltiger Prozess ist, der sich über Generationen hinweg erstreckt und auswirkt. So scheint die Identifikation von Zuwandererfamilien sowohl mit dem Bestimmungs- als auch mit dem Herkunftsland für den Bildungserfolg der nächsten Generation von Bedeutung zu sein. Dabei stellt eine hohe Verbundenheit mit der ethnischen Gruppe keinesfalls ein Bildungshindernis dar, sondern kann im Gegenteil dem Bildungserfolg zuträglich sein. Insbesondere Mütter sollten jedoch in ihren Integrationsbemühungen unterstützt werden, da Strategien zur außerschulischen Förderung ihrer Kinder (z.B. Hausaufgabenbetreuung oder Kontakt mit Lehrern) dadurch an Effizienz gewinnen können. Die genauen Mechanismen, über die elterliche ethnische Identitäten den Bildungserfolg der nächsten Generation beeinflussen, muss jedoch zum jetzigen Zeitpunkt eine offene Frage bleiben.

Im Prozess der Arbeitsplatzsuche erscheint insbesondere der Reservationslohn als Entscheidungsvariable etwa durch Referenzgruppen und Risikopräferenzen beeinflusst, die sich zudem zwischen den Einwanderergenerationen und im Vergleich mit Einheimischen unterscheiden. Auch vor dem Hintergrund, dass der Prozess der Arbeitsplatzsuche in $\mathrm{Zu}-$ kunft wohl weiter an Bedeutung zunehmen wird, zieht dies wiederum wirtschaftspolitische Implikationen nach sich. Es bieten sich zum Beispiel viel versprechende Ansatzpunkte für eine entsprechende Ausrichtung der aktiven Arbeitsmarktpolitik wie etwa spezielle Maßnahmen für die Gruppe von Arbeitssuchenden mit geringer Risikoaversion (z.B. Monitoring ihrer Suchanstrengungen) an. Weiterführende Studien, die die genutzten Suchkanäle und unternommenen Suchanstrengungen der Arbeitslosen untersuchen, können in diesem Zusammenhang Antworten auf bislang noch offene Fragen liefern. So haben sich informelle Suchkanäle 
über ethnische Netzwerke, Familien und Freunde als erfolgreich erwiesen. Diese greifen jedoch in hohem Maße auf soziale Netzwerke zurück, die sich zwischen Einwanderern und Einheimischen, aber auch innerhalb der Gruppe von Immigranten erheblich unterscheiden können. Es ist auch zu vermuten, dass sich ethnische Identitäten in diesem $\mathrm{Zu}$ sammenhang positiv auswirken.

Ethnische Vielfalt birgt ein substanzielles ökonomisches Potenzial für den wirtschaftlichen Erfolg von Individuen, das bisher noch $\mathrm{zu}$ wenig verstanden und ausgeschöpft wird. Dabei geht es nicht darum, dass ein buntes Nebeneinander verschiedener Kulturen und Lebensweisen existiert und Immigranten und Einheimische nicht aufeinander zugehen. Dieses Verständnis eines multikulturellen Ansatzes ist längst überholt und mit dem Konzept der Integration nicht vereinbar.

Bisherige Forschungsergebnisse sowie die Ergebnisse des Projektes zeigen, dass kulturelle Vielfalt in Form einer relativ stärkeren ethnischen Identität auch auf individueller Ebene ökonomischen Erfolg nicht hindert, sondern im Gegenteil positive Auswirkungen haben kann. Es sollte von der Mehrheitsgesellschaft daher nicht gefordert werden, dass sich Zuwanderer assimilieren, vielmehr sollte das ethnischspezifische Humankapital von Einwanderern als Zugewinn und Vorteil anerkannt werden. Dies setzt voraus, dass Einheimische und Zuwanderer aufeinander zugehen. Es geht also darum, in beide Kulturen - die des Heimat- und des Gastlandes - einzutauchen und ein wechselseitiges Verständnis zu entwickeln.

Dies hat 2010 die deutsche Nationalmannschaft bei der Fußball-Weltmeisterschaft in Südafrika beispielhaft verdeutlicht: Aus der ethnischen Vielfalt der ausgesuchten Spieler entstand hier zunächst ein äußerst attraktives Produkt. Dennoch war es möglich, eine hohe nationale Identifikation der Mannschaft wie der Nation mit der Mannschaft zu erreichen. Nationale Identität ist mit einer ethnischen Vielfalt in der Nation gut vereinbar. Dies gilt auch für die Wirtschaft, der es jedoch gelingen muss, die natürlichen Vorteile einer multi-ethnisch integrierten Gesellschaft besser für ihre Produktionsprozesse zu nutzen.

\section{Executive summary}

Whereas the social and labor market integration of ethnic minorities is recognized as a major challenge in many Western European countries, the economic potential of cultural diversity is often underestimated. Previous research has documented the central role of attitudes, perceptions, and in particular ethnic identity in this context. Against this background, the present article summarizes a number of recent studies which extend this line of research in two important aspects. First, the question is addressed whether immigrants' cultural integration has a long-term effect on economic outcomes of subsequent generations. Specifically the group of second generation migrants is more and more a concern, both from an academic and a policy perspective. In the course of the past century, many Western European countries have accumulated sizeable stocks of migrants and their descendants. Although one would expect migrant-native differences in economic outcomes to decrease substantially or even vanish from one generation to the next, this is not necessarily the case. Second, the process of job search is analyzed by incorporating concepts of ethnic identity and by distinguishing between first and second generation migrants. Since employment biographies become more unstable and fragmented, and labor markets in general more flexible, the importance of job search efficiency increases. However, there may be crucial differences in the job search behavior between first and second generation migrants, which in turn might be related to their ethnic identification. One major finding of the studies discussed in this paper is that ethnic identity has a persistent effect on economic outcomes, i.e., the effect of ethnic identity materializes over migrant generations. Other important findings can be summarized as follows. First, a process of cultural integration over migrant generations occurs for all ethnic minority groups in Germany. There are for example no major differences between natives and second generation migrants with regard to marriage decisions, fertility, or political interests. However, the intergenerational process of cultural integration appears to be somewhat slower for particular ethnic groups with an a priori higher cultural distance from the majority culture in the host country. Second, parental ethnic identity matters for the educational outcomes of second generation migrants. The identification of their parents with both the host country and the country of origin has an effect on immigrant children's educational success. Interestingly, a rather strong identification with the country of origin does not appear to be an obstacle for a successful educational career. However, mothers' integration efforts with the host country should be encouraged. The results generally point at the supposition that for immigrant children, growing up in integrated, rather than separated or assimilated families might be conductive for educational success. Third, in particular reservation wages of unemployed immigrant job seekers appear to be influenced by reference groups and risk preferences, which moreover differ between migrant generations and also in comparison with natives. These findings offer interesting perspectives, e.g., with regard to the design and targeting of active labor market policy. It may for example be reasonable to specifically focus on less risk averse individuals with measures such as job search requirements and monitoring, which potentially lower the expectations and reservation wages of those unemployed individuals. Overall, the economy can gain productivity and efficiency by recognizing and incorporating multiethnic factors. Cultural assimilation that goes along with a 
loss of migrants' own cultural heritage does not appear to be the sole or dominant strategy of an economically successful integration. In contrast, ethnic human capital may even represent an interesting complementary factor that can be beneficial for economic success. To tap the full potential of ethnic and cultural diversity, an increased cultural and ethnic open-mindedness of the migrant as well as the native population is desirable.

Danksagung Die Autoren danken der Deutschen Forschungsgemeinschaft (DFG) für die finanzielle Förderung des Projektes Ethnic Diversity and Labor Market Success im Rahmen des Schwerpunktprogramms „Flexibilisierungspotentiale bei heterogenen Arbeitsmärkten“. Dieses Projekt ist, da zu einem späteren Zeitpunkt begonnen, noch nicht abgeschlossen. Insofern reflektiert der vorliegende Artikel einen Zwischenstand der bisherigen Forschungsergebnisse.

\section{Literatur}

Akerlof, G.A., Kranton, R.E.: Economics and identity. Q. J. Econ. 115(3), 715-753 (2000)

Alesina, A., La Ferrara, E.: Ethnic diversity and economic performance. J. Econ. Lit. 43(3), 762-800 (2005)

Algan, Y., Dustmann, C., Glitz, A., Manning, A.: The economic situation of first- and second-generation immigrants in France, Germany, and the United Kingdom. Econ. J. (Lond.) 120(542), 4-30 (2010)

Audretsch, D.B., Dohse, D., Niebuhr, A.: Cultural diversity and entrepreneurship: a regional analysis for Germany. CEPR discussion paper No. 6945 (2008)

Bellini, E., Ottaviano, G.I.P., Pinelli, D., Prarolo, G.: Cultural diversity and economic performance: evidence from European regions. FEEM DP No. 632009 (2009)

Bisin, A., Verdier, T.: The economics of cultural transmission and the dynamics of preferences. J. Econ. Theory 97(2), 298-319 (2001)

Borghans, L., Duckworth, A.L., Heckman, J.J., ter Weel, B.: The economics and psychology of personality traits. J. Hum. Resour. 43(4), 972-1059 (2008)

Caliendo, M., Falk, A., Kaiser, L.C., Schneider, H., Uhlendorff, A., van den Berg, G.J., Zimmermann, K.F.: The IZA evaluation data set: towards evidence-based labor policy-making. IZA discussion paper 5400, Institute for the Study of Labor (IZA), Bonn (2010)

Constant, A.F., Zimmermann, K.F.: Measuring ethnic identity and its impact on economic behavior. J. Eur. Econ. Assoc. 6(2-3), 424433 (2008)

Constant, A.F., Zimmermann, K.F.: Work and money: payoffs by ethnic identity and gender. Res. Labor Econ. 29, 3-30 (2009)

Constant, A.F., Zimmermann, K.F.: Migration, ethnicity and economic integration. In: Jovanovic, M.N. (Hrsg.) International Handbook on the Economics of Integration, Bd. III, S. 145-168. Edward Elgar, Cheltenham (2011, forthcoming)

Constant, A.F., Gataullina, L., Zimmermann, K.F.: Ethnosizing immigrants. J. Econ. Behav. Organ. 69(3), 274-287 (2009a)

Constant, A.F., Kahanec, M., Rinne, U., Zimmermann, K.F.: Ethnicity, job search and labor market reintegration of the unemployed. IZA discussion paper 4660, Institute for the Study of Labor (IZA), Bonn (2009b)

Constant, A.F., Roberts, R., Zimmermann, K.F.: Ethnic identity and immigrant homeownership. Urban Stud. 46, 1879-1898 (2009c)

Constant, A.F., Krause, A., Rinne, U., Zimmermann, K.F.: Reservation wages of first and second generation migrants. IZA discussion paper 5396, Institute for the Study of Labor (IZA), Bonn (2010a)
Constant, A.F., Krause, A., Rinne, U., Zimmermann, K.F.: Economic preferences and attitudes of the unemployed: are natives and second generation migrants alike? IZA discussion paper 5380, Institute for the Study of Labor (IZA), Bonn (2010b)

Constant, A.F., Nottmeyer, O., Zimmermann, K.F.: Cultural integration in Germany. In: Bisin, A., Manning, A., Verdier, T. (Hrsg.) Cultural integration in Europe. Oxford University Press, Oxford (2011, forthcoming)

Dustmann, C., Fabbri, F.: Language proficiency and labour market performance of immigrants in the UK. Econ. J. (Lond.) 113(489), 695-717 (2003)

Eichhorst, W., Kuhn, A., Thode, E., Zenker, R.: Traditionelle Beschäftigungsverhältnisse im Wandel. IZA research report 23, Institute for the Study of Labor (IZA) (2010)

Heath, A.F., Li, Y.: Period, life-cycle and generational effects on ethnic minorities. Köln. Z. Soziol. Soz.psychol., Sonderh. 48, 277-306 (2008)

Kaas, L., Manger, C.: Ethnic discrimination in Germany's labour market: a field experiment. IZA discussion paper 4741, Institute for the Study of Labor (IZA), Bonn (2010)

Kahanec, M., Zaiceva, A.: Labor market outcomes of immigrants and non-citizens in the EU: an East-West comparison. Int. J. Manpow. 30(1/2), 97-115 (2009)

Kahanec, M., Zimmermann, K.F.: Migration in an enlarged EU: a challenging solution? In: Keereman, F., Szekely, I. (Hrsg.) Five Years of an Enlarged EU-A Positive Sum Game, S. 63-94. Springer, Berlin (2010)

Kalter, F., Granato, N.: Educational hurdles on the way to structural assimilation in Germany. In: Heath, A.F., Cheung, S.Y. (Hrsg.) Unequal Chances: Ethnic Minorities in Western Labour Markets, S. 271-319 (2007)

Krause, A., Rinne, U., Schüller, S.: Kick it like özil? Second generation migrants' initial disadvantage and the failure of the education system. Working paper, Institute for the Study of Labor (IZA), Bonn (2010)

Ottaviano, G.I.P., Peri, G.: The economic value of cultural diversity: evidence from US cities. J. Econ. Geogr. 6(1), 9-44 (2006)

Pannenberg, M.: Risk attitudes and reservation wages of unemployed workers: evidence from panel data. Econ. Lett. 106(3), 223-226 (2010)

Riphahn, R.: Are there diverging time trends in the educational attainment of nationals and second generation immigrants? Jahrb. Natl.ökon. Stat. 225(3), 325-346 (2005)

Rühl, S.: Grunddaten der Zuwanderungsbevölkerung in Deutschland. Working paper 27, Bundesamt für Migration und Flüchtlinge (2009)

Schüller, S.: Ethnic identity and educational attainment of second generation immigrants in Germany. Working paper, Deutsches Institut für Wirtschaftsforschung (DIW Berlin), Berlin (2010)

Tajifel, H.: Human Groups and Social Categories. Cambridge University Press, Cambridge (1981)

Ulf Rinne studierte Volkswirtschaftslehre in Bonn und Lissabon. Er wurde im Juli 2009 von der Freien Universität Berlin promoviert. Er ist seit August 2005 am IZA Bonn tätig, zunächst als Resident Research Affiliate und seit Januar 2009 als Research Associate und Persönlicher Referent des Direktors. Seine Forschungsschwerpunkte liegen in der empirischen Arbeitsmarktforschung, insbesondere in den Bereichen Migration, Humankapital und Bildung.

Simone Schüller studierte Volkswirtschaftslehre in Mainz, Pisa und Leipzig und schloss ihr Studium im August 2007 mit dem Diplom ab. Von 2007 bis 2009 nahm sie am Young Graduate Trainee Programm der Europäischen Raumfahrtagentur teil. Seit September 2009 ist Simone Schüller am DIW Berlin als wissenschaftliche Mitarbeiterin tätig. Ihre aktuellen Forschungsschwerpunkte liegen in der empirischen 
Arbeitsmarkt- und Migrationsforschung, der angewandten Mikroökonometrie sowie der Glücksforschung.

Klaus F. Zimmermann ist Professor für Wirtschaftliche Staatswissenschaften der Universität Bonn, Direktor des IZA Bonn, Präsident des DIW Berlin, Honorarprofessor für Volkswirtschaftslehre der Freien Universität Berlin, Honorarprofessor der Renmin University of China in Peking und Vorsitzender der Arbeitsgemeinschaft deutscher wirtschaftswissenschaftlicher Forschungsinstitute (ARGE). Seine Forschungsinteressen liegen in den Bereichen Arbeitsökonomie, Bevölke- rungsökonomie, Migration, Industrieökonomie und Ökonometrie. Er ist Mitglied der Deutschen Akademie der Naturforscher Leopoldina, des World Economic Forum's Global Agenda Council on Migration und der Akademia Europaea. Er hat außerdem zahlreiche nationale Regierungen und die Europäische Kommission beraten sowie bislang 37 Bücher verfasst oder herausgegeben, 103 Forschungsarbeiten in referierten Fachzeitschriften publiziert und 124 Kapitel in Sammelbänden geschrieben. 